\title{
TOEPLITZ OPERATORS AND ALGEBRAS OF BOUNDED ANALYTIC FUNCTIONS ON THE DISK
}

\author{
by R. C. SMITH
}

(Received 22 December, 1989)

1. Introduction. Here and throughout, $A$ is a closed subalgebra of $H^{\infty}$ that contains the disk algebra and $M(A)$ denotes the maximal ideal space of $A$. Because $A$ contains the function $f_{0}(z)=z$, we can define the fiber $M_{\lambda}(A)$ of $M(A)$ for $\lambda \in \partial D$ (the unit circle) in the usual way; i.e., $M_{\lambda}(A)=\left\{\phi \in M(A): f_{0}(\phi)=\lambda\right\}$. The Bergman space $L_{a}^{2}(D)$ of the unit disk $D$ is the $L^{2}(D, d x d y)$-closure of $A$. Let $P: L^{2}(D, d x d y) \rightarrow L_{a}^{2}(D)$ be the orthogonal projection. For $f \in L^{\infty}(D, d x d y)$, define the multiplication operator $M_{f}: L^{2}(D, d x d y) \rightarrow L^{2}(D, d x d y)$ by

$$
M_{f} g=f g, \quad g \in L^{2}(D, d x d y)
$$

and define the Toeplitz operator $T_{f}: L_{a}^{2}(D) \rightarrow L_{a}^{2}(D)$ by

$$
T_{f} g=P M_{f} g, \quad g \in L_{a}^{2}(D) .
$$

Let $T(A)$ be the $C^{*}$-algebra of bounded operators on $L_{a}^{2}(D)$ generated by $\left\{T_{f}: f \in\right.$ $C(M(A))\}$ and let $C(A)$ be the commutator ideal of $T(A)$. Denote the maximal ideal space of $T(A) / C(A)$ by $E(A)$. The McDonald-Sundberg theorem ([8]) asserts that $E\left(H^{\infty}\right)$ consists of the one point Gleason parts of $M\left(H^{\infty}\right)$. At the other extreme, if $A$ is the disk algebra, then $E(A)$ is the unit circle by a theorem of Coburn ([2]). The unit circle consists of the one point Gleason parts of $M(A)$ if $A$ is the disk algebra, so a natural question arises: does $E(A)$ always consist of the one point Gleason parts of $M(A)$ ? As we see below, the answer to this question is no. However, we can see that $E(A)$ consists of the one point Gleason parts of $M(A)$ when $A=H^{\infty} \cap C(D \cup K)$, where $K$ is a closed set in $\partial D$. Thus there is a class of algebras (albeit of somewhat limited interest) that includes the disk algebra and $H^{\infty}$ allows us to "interpolate" between Coburn's theorem and the McDonald-Sundberg theorem.

For any unexplained notions from the theory of function algebras (e.g., maximal ideal space, Gleason parts, Shilov boundary) see Gamelin's book [5].

2. Sundberg's criterion and applications. For $f \in A$ and $g \in L^{\infty}(D, d x d y)$ we can easily see that $T_{\tilde{f}_{g}}=T_{\bar{f}} T_{g}$ and $T_{f g}=T_{g} T_{f}$. By an argument in Chapter 7 of [4], the commutator ideal $C(A)$ coincides with the semicommutator ideal of $T(A)$. This allows us to use the main result of [11] to assert that $E(A)=\{\phi \in M(A): f \in C(M(A))$ with $f(\phi)=0$ implies $M_{f}$ is not bounded below on $\left.L_{a}^{2}(D)\right\}$. As a consequence of his proof, Sundberg obtains the spectral inclusion $f(E(A)) \subset \sigma\left(T_{f}\right)$ for $f \in C(M(A))$ without any ancillary work and so each theorem identifying $E(A)$ has an immediate corollary giving a spectral inclusion result. This criterion of Sundberg for membership in $E(A)$ is the crucial ingredient in what follows. Indeed, part of the purpose of this note is to display the utility of Sundberg's criterion.

Let $R: M\left(H^{\infty}\right) \rightarrow M(A)$ be the restriction map $R \phi=\phi \mid A, \phi \in M\left(H^{\infty}\right)$.

THEOREM 2.1. $R\left(E\left(H^{\infty}\right)\right)=E(A)$. 
Proof. Let $\phi \in E\left(H^{\infty}\right)$ and suppose $f \in C(M(A))$ with $f(R \phi)=0$. Then $f \circ R \in$ $C\left(M\left(H^{\infty}\right)\right)$ and $f \circ R$ vanishes at $\phi$ whereby $M_{f \circ R}$ is not bounded below on $L_{a}^{2}(D)$. Because $f=f \circ R$ on $D, M_{f}=M_{f \circ R}$ and so $R \phi \in E(A)$.

For the other inclusion, suppose $\phi \in M(A)$ does not belong to $R\left(E\left(H^{\infty}\right)\right)$. Clearly $E(A)$ is contained in the $M(A)$-closure of $D$ and so without loss of generality, assume $\phi$ lies in the $M(A)$-closure of $D$. There are disjoint open sets $W, V$ in $M(A)$ such that $\phi \in W$ and $R\left(E\left(H^{\infty}\right)\right) \subset V$. Now $R^{-1}(W)$ and $R^{-1}(V)$ are disjoint open sets in $M\left(H^{\infty}\right)$ and $W \cap D=R^{-1}(W) \cap D$ so $U=W \cap D$ is an open set in $D$ whose $M\left(H^{\infty}\right)$-closure does not meet $E\left(H^{\infty}\right)$. By exercise 2 from Chapter $\mathrm{X}$ of [6], there is a finite union $S$ of interpolating sequences such that $U \subset\left\{z \in D: \rho(z, w)<\frac{1}{2}\right.$ for some $\left.w \in S\right\}$, where

$$
\rho(z, w)=\left|\frac{z-w}{1-\bar{w} z}\right| .
$$

By [7], it follows that there is some $C>0$ such that

$$
\int_{D \backslash U}|g(z)|^{2} d x d y \geq C\|g\|^{2}, \quad z=x+i y
$$

for all $g \in L_{a}^{2}(D)$. Take $f \in C(M(A))$ with $f(\phi)=0$ and $f \equiv 1$ off $W$. Then

$$
\left\|M_{f} g\right\|^{2} \geq \int_{D \backslash U}|g(z)|^{2} d x d y \geq C\|g\|^{2}
$$

for $g \in L_{a}^{2}(D)$. By Sundberg's Criterion, $\phi \notin E(A)$.

This theorem characterizes the set $E(A)$, but is unsatisfying in comparison to Coburn's theorem and the McDonald-Sundberg theorem which characterize $E(A)$ (for $A=$ disk algebra or $H^{\infty}$ ) in terms of a natural notion from the theory of function algebras. Such a result does not seem possible in our context. Example 2.2 shows that $E(A)$ does not have to'consist of one point Gleason parts of $M(A)$ and the other examples point out more subtle phenomena.

EXAMPLE 2.2. Let $u$ be an inner function not in the disk algebra and let $A$ be the algebra generated by the disk algebra and $u$. Suppose $\left\{\psi_{0}\right\}$ is a one point Gleason part of $M\left(H^{\infty}\right)$ and that $u\left(\psi_{0}\right)=0$. Then $R \psi_{0}$ belongs to a nontrivial Gleason part of $M(A)$. Thus $E(A)$ does not consist of one point Gleason parts of $M(A)$.

Proof. Let $\rho(\phi, \psi)=\sup \left\{|f(\phi)|: f \in A,\|f\|_{\infty} \leq 1\right.$ and $\left.f(\psi)=0\right\}$ denote the pseudohyperbolic distance on $M(A)$. We want to find $\phi \in M(A)$ such that $\phi \neq R \psi_{0}$ and $\rho\left(\phi, R \psi_{0}\right)<1$. Let $\lambda \in \partial D$ such that $\psi_{0} \in M_{\lambda}\left(H^{\infty}\right)$ and take $\phi \in M_{\lambda}\left(H^{\infty}\right)$ such that $0<|u(\phi)|<1$. This can be done because the cluster set of $u$ at $\lambda$ is the closed unit disk. Now $A$ contains a dense set of elements of the form $F=g+f p(u)$ where $f$ and $g$ belong to the disk algebra and $p$ is a polynomial with $p(0)=0$ and $\|p\|_{\infty}=1$. Suppose $F\left(R \psi_{0}\right)=0$. Then $0=F\left(R \psi_{0}\right)=g(\lambda)+f(\lambda) p\left(u\left(\psi_{0}\right)\right)=g(\lambda)$ so $F=f p(u)$ on $M_{\lambda}(A)$. Thus $|f(\lambda)| \leq$ $\|F\|_{\infty}$ and taking $\|F\|_{\infty} \leq 1$, we get

$$
|F(R \phi)|=|f(\lambda)||p(u(\phi))| \leq|p(u(\phi))| \leq|u(\phi)|
$$

by Schwarz's Lemma. It follows that $\rho\left(R \phi, R \psi_{0}\right)=|u(\phi)|<1$; i.e., $R \phi \neq R \psi_{0}$ belongs to the same Gleason part as $R \psi_{0}$. The McDonald-Sundberg theorem and our Theorem 2.1 imply $R \psi_{0} \in E(A)$. 
Example 2.3. Let $A$ be the algebra from example 2.2 with

$$
u(z)=\exp \frac{z+1}{z-1} .
$$

Then $E(A)=(\partial D \backslash\{1\}) \cup E_{1}(A)$ where $E_{1}(A)=M_{1}(A) \cap E(A)$ consists of those $\phi \epsilon$ $M_{1}(A)$ such that $|u(\phi)|=1$ or 0 .

Proof. If $|u(\phi)|=1$ then $\phi \in \partial(A)$, the Shilov boundary of $A$, so $\phi \in E(A)$ by ([10]). By Theorem 2.1, if $\phi_{0} \in M_{1}(A) \cap R\left(E\left(H^{\infty}\right)\right.$ ), then $\phi_{0} \in E(A)$. It is well known (see Chapter $\mathrm{X}$ of [6]) that if $u$ is a singular inner function and $\psi \in M\left(H^{\infty}\right)$ satisfies $u(\psi)=0$, then $\{\psi\}$ is a Gleason part of $M\left(H^{\infty}\right)$. Thus $\phi_{0} \in M_{1}(A)$ with $u\left(\phi_{0}\right)=0$ implies $\phi_{0} \in E(A)$, as in Example 2.2. We can say more here; namely $E(A)=\partial(A) \cup\left\{\phi_{0}\right\}$. To show this, we need the observation by A. Matheson that

$$
b(z)=\frac{u(z)-w}{1-\bar{w} u(z)}
$$

is an interpolating Blaschke product for all $w \in D \backslash\{0\}$. Because this result is unpublished, we sketch a proof. The function $b$ extends analytically across $\partial D \backslash\{1\}$ and so the singular factor of $b$ must be of the form

$$
S(z)=\exp t \frac{z+1}{z-1}
$$

for some $t \geq 0$. If $t>0$, then $|S(z)| \rightarrow 0$ as $z$ tends radially to 1 . But $b(z) \rightarrow-w \neq 0$ as $z$ tends radially to 1 and so $t=0$; i.e., $b$ is a Blaschke product. To see that the zero sequence of $b$ is an interpolating sequence, consider the conformal map $f(\zeta)=$ $(\zeta-i) /(\zeta+i)$ from the upper half plane to the unit disk. Then

$$
(b \circ f)^{-1}(\{w\})=\left\{\zeta \in \mathbb{C}: \operatorname{Im} \zeta=\log \frac{1}{|w|} \text { and } \operatorname{Re} \zeta=\arg w+2 n \pi, n \text { an integer }\right\}
$$

which is easily seen to be an interpolating sequence for the upper half plane and it follows that $b^{-1}(\{w\})$ is an interpolating sequence in $D$. Now $M_{b}$ is bounded below on $L_{a}^{2}(D)$ ([8]). Thus if $\phi \in M_{1}(A)$ and $u(\phi)=w \in D \backslash\{0\}$, then $\phi \notin E(A)$.

EXAMPLE 2.4. Let $u$ be an inner function belonging to the little Bloch space. That is, $\lim _{\mid z \mapsto 1}\left(1-|z|^{2}\right)\left|u^{\prime}(z)\right|=0$. Then

$$
b(z)=\frac{u(z)-w}{1-\bar{w} u(z)}
$$

belongs to the little Bloch space for all $w \in D$. Such a function $b$ cannot be a finite product of interpolating Blaschke products ([6, Chapter X, Exercise 11]) and, by [8], it follows that there is some $\phi \in E\left(H^{\infty}\right)$ such that $b(\phi)=0$. Let $A$ be the algebra generated by the disk algebra and $u$. Then $E(A)=M(A) \backslash D$ by Theorem 2.1.

Note that the following algebra was studied by Dawson [3], who showed that $D$ is not dense in $M(A)$.

EXAMPLE 2.5. (The Gramophone algebra) Let $A$ be the algebra generated by the disk algebra and the outer function $G(z)=(1-z)^{i}$ where we take $-(\pi / 2)<\arg (1-z)<\pi / 2$. Then $E(A)=\partial(A)$. 
Proof. The cluster set of $G$ at 1 is $\left\{z \in \mathbb{C}: e^{-\pi / 2} \leq|z| \leq e^{\pi / 2}\right\}$. If $\phi \in M_{1}(A)$ and $|G(\phi)|<e^{-\pi / 2}$, then $\phi$ lies outside the $M(A)$-closure of $D$ and so $\phi \notin E(A)$. Suppose $\phi \in M_{1}(A)$ with $e^{-\pi / 2}<|G(\phi)|<e^{\pi / 2}$. Now the level sets of $|G|$ in $D$ are secants terminating at 1 and so $\phi$ lies in the $M(A)$-closure of the interior of a Stolz angle at 1 . Let $U$ be a truncation (so that $U \cap \partial D=\{1\}$ ) of this region. By [7], there is some $C>0$ such that

$$
\int_{D \backslash U}|g(z)|^{2} d x d y \geq C\|g\|^{2} \text { for } g \in L_{a}^{2}(D) .
$$

As in the proof of Theorem 2.1 we see that $\phi \notin E(A)$. Now $\partial(A)=(\partial D \backslash\{1\}) \cup$ $\left\{\phi \in M_{1}(A):|G(\phi)|=e^{ \pm \pi / 2}\right\}$ and so $E(A)=\partial(A)$ by [10]. It is worth noting that $\phi \in M_{1}(A)$ and $|G(\phi)|=e^{-\pi / 2}$ imply that $\phi$ lies in a nontrivial Gleason part of $M(A)$.

Let $K$ be a closed set in $\partial D$ and let $A=H^{\infty} \cap C(D \cup K)$, the algebra of bounded analytic functions on $D$ that extend continuously to $K$. For $\lambda \in K$ we clearly get $M_{\lambda}(A)=\{\lambda\}$. Suppose $\lambda \notin K$. Take a closed arc $\Gamma$ in $\partial D$ centered at $\lambda$ such that $\Gamma \cap K=\varnothing$. There is a conformal map $h$ of $D$ onto a domain in $D$ such that $h(\Gamma)$ is an arc in $\partial D$ with $h(\lambda)=\lambda, h(K) \subset D$ and such that $h$ extends continuusly to $D([9$, Chapter V, Section 7]). Given $f \in H^{\infty}, g=f \circ h$ belongs to $A$ and $\lim _{z \rightarrow \lambda|z|<1} \sup _{|z|}|f(z)-g(z)|=0$. Thus $A\left|M_{\lambda}\left(H^{\infty}\right)=H^{\infty}\right| M_{\lambda}\left(H^{\infty}\right)$ and $R \mid M_{\lambda}\left(H^{\infty}\right)$ is the identity map. Put $E_{\lambda}\left(H^{\infty}\right)=E\left(H^{\infty}\right) \cap$ $M_{\lambda}\left(H^{\infty}\right)$. Applying Theorem 2.1 we obtain the following.

TheOREM 2.6. Let $K$ be a closed set in $\partial D$ and let $A=H^{\infty} \cap C(D \cup K)$. Then $E(A)=\left(\bigcup_{\lambda \notin K} E_{\lambda}\left(H^{\infty}\right)\right) \cup K$. In particular, $E(A)$ consists of the one point Gleason parts of
$M(A)$.

Remarks. Any of the examples 2.2-2.5 suffices to show that $E(A)$ does not necessarily consist of one point Gleason parts of $M(A)$. In fact, in examples 2.2-2.4, $E(A)$ is not even contained in the closure of the one point Gleason parts. Of course, the pseudohyperbolic distance from $R \phi$ to $R \psi$ in $M(A)$ is no larger than the pseudohyperbolic distance from $\phi$ to $\psi$ in $M\left(H^{\infty}\right)$ whence $E(A)$ contains the one point Gleason parts of $M(A)$ that lie in the $M(A)$-closure of $D$.

In examples $2.2-2.4$, we see the restriction map $R$ sending one point Gleason parts of $M\left(H^{\infty}\right)$ to points belonging to nontrivial parts of $M(A)$, but in each of $2.2-2.4$, we have $R\left(\partial\left(H^{\infty}\right)\right)=\partial(A)$ which consists of peak points for these algebras. However, in example 2.5 we see that $R\left(\partial\left(H^{\infty}\right)\right.$ ) can meet a nontrivial Gleason part of $M(A)$. The first example (2.2) shows that Theorem 2.6 does not directly generalize to the algebras considered by Chang and Marshall in [1] and examples 2.3 and 2.4 serve to point out the difficulty of formulating a nice result in this context. To be more specific, we see that $E(A)$ can be as small (example 2.3) or as large (example 2.4) as is allowed by Theorem 2.1 and example 2.2.

Sundberg shows that $E(A)$ is the largest set in $M(A)$ such that $f(E(A)) \subset \sigma\left(T_{f}\right)$ for all $f \in C(M(A))([11])$. Because $T_{z}^{*} T_{z}-T_{z} T_{z}^{*}$ is a compact operator on $L_{a}^{2}(D)$ (see [8]) and the compact operators form a minimal closed two-sided ideal in the bounded operators ([4]), it follows that $C(A)$ contains the compact operators on $L_{a}^{2}(D)$. From standard facts about the spectrum $([4])$, we obtain $f(E(A)) \subset \sigma_{e}\left(T_{f}\right)$ for all $f \in C(M(A))$. 
If we define our operators on the Hardy space $H^{2}$ of the unit circle instead of the Bergman space, then $\left(\left[4\right.\right.$, Chapter 7]) the sets $E(A)$ in question turn out to be $\partial\left(H^{\infty}\right)$ when $A=H^{\infty}$ and $\partial D=\partial(A)$ when $A$ is the disk algebra. This Hardy space case is better behaved than the situation for the Bergman space. In fact $([\mathbf{1 0}])$, if $\mu$ is a probability measure with supp $\mu=\partial(A)$ then $\partial(A)=E(A)=\{\phi \in M(A): f \in C(M(A))$ with $f(\phi)=0$ implies $M_{f}$ is not bounded below on the $L^{2}(\mu)$-closure of $\left.A\right\}$.

Acknowledgements. The author is indebted to Alec Matheson for the result used in example 2.3 and, in particular, the author would like to thank him for pointing out that the argument is simplified greatly by passing to the upper half plane. Thanks are also due to Len Miller for several discussions on these topics. Finally, the author would like to thank Sheldon Axler and Brian Cole for expressing an interest in some of these questions.

\section{REFERENCES}

1. S. Y. Chang and D. Marshall, Some algebras of bounded analytic functions containing the disk algebra, in Banach spaces of analytic functions, Lecture Notes in Math. 604, Springer Verlag, 1977.

2. L. Coburn, Singular integral operators and Toeplitz operators on odd spheres, Indiana Univ. Math. J. 23, (1973), 433-439.

3. D. Dawson, Subalgebras of $H^{\infty}$, Ph.D. Thesis, Indiana University, 1975.

4. R. Douglas, Banach algebra techniques in operator theory (Academic Press, 1972).

5. T. Gamelin, Uniform Algebras (Prentice-Hall, 1969).

6. J. Garnett, Bounded Analytic Functions (Academic Press, 1981).

7. D. Luecking, Inequalities on Bergman spaces, Illinois J. Math. 25 (1981), 1-11.

8. G. McDonald and C. Sundberg, Toeplitz operators on the disk, Indiana Univ. Math. J. 28 (1979), 595-611.

9. Z. Nehari, Conformal Mapping (McGraw-Hill, 1952).

129-131. C. Sundberg, Exact sequences for generalized Toeplitz operators, Proc. Amer. Math. Soc.

10. R. Smith, Toeplitz operators on abstract Hardy spaces, Glasgow Math. J. 30 (1988), 101 (1987), 634-636.

Department of Mathematics and Statistics

MisSISSIPPI STATE UNIVERSITY

MissisSIPPI 39762

USA 